\title{
CONTINUOS MONITORING OF LIQUID WATER CLOUDS AND AEROSOLS WITH DUAL-FOV LIDAR POLARIZATION TECHNIQUE
}

\author{
Cristofer Jimenez $^{1 *}$, Albert Ansmann ${ }^{1}$, Ronny Engelmann ${ }^{1}$, Patric Seifert ${ }^{1}$, Robert Wiesen ${ }^{1}$, Martin \\ Radenz $^{1}$, and Ulla Wandinger ${ }^{1}$ \\ ${ }^{1}$ Leibniz Institute for Tropospheric Research, Permoserstr. 15, 04318 Leipzig, Germany \\ *Email: cristofer.jimenez@tropos.de
}

\begin{abstract}
In this work we evaluate the possibilities to assess aerosol-cloud interactions in short time scales (2 min.) on an observational base. Retrievals of the cloud effective radius and number concentration in a liquid-water cloud by using the multiple scattering technique Dual-FOV Polarization lidar, together with the aerosol extinction coefficient in the boundary layer has shown a correspondence between the aerosol and cloud properties in the 6 hours case presented, obtaining a value of $A C I_{N}=$ $0.76 \pm 0.29$, which corroborates the potential of lidar observations to study the relation between aerosols and clouds on small scales.
\end{abstract}

\section{INTRODUCTION}

Atmospheric aerosols have been on the hotspot of climate research in the last decades due to their influence on the transfer of radiant energy and distribution of latent heat through the atmosphere. Directly through the absorption, emission and scattering of solar and terrestrial radiation and indirectly, acting as cloud condensation nuclei (CCN) and as ice nucleating particle (INP) in water clouds. To better understand the influence of these particles in weather and a changing climate, observational evidence of the aerosols playing his role in clouds is needed. For this reason, several techniques for the monitoring and characterization of aerosols and clouds, have emerged in the last years.

In the case of liquid-phase clouds, a metric to aerosols and clouds is the so called aerosol-cloud interaction index, defined as the variation of the cloud droplet number concentration (CDNC), in the cloud base region at a constant liquid water content, divided the variation of the aerosol extinction $\sigma_{\text {aer }}$ (or alternatively the $\mathrm{CCN}$ concentration) [1].

$A C I_{N}=\frac{\Delta \ln C D N C}{\Delta \ln \sigma_{a e r}}$
Lidar systems have proved to have great potential for the assessment of this index, allowing estimations of $\mathrm{CCN}$ concentrations, from measurements of the aerosol extinction coefficient and depolarization in the non-saturated region below cloud base [2]. Lidar systems can also provide information about the cloud optical and microphysical properties, which is contained in the lidar returns affected by multiple scattering (MS) in water clouds [3]. The Dual Field-of-View Raman (DFOV-Raman) technique, implemented on the MARTHA system (Multiwavelength Tropospheric Raman lidar for Temperature, Humidity, and Aerosol profiling) at TROPOS, permits non-ambiguous retrievals of droplet extinction and effective size profiles in the cloud base region by means of the MS-affected Raman return at two FOVs [4]. The technique needs however stable cloud base layers and can only be applied during nighttime. In order to decrease the time resolution and perform observations also during daytime, a Dual-FOV Polarization method (DFOV-Pol) was developed and proposed, which by measuring elastic cross- and co-polarized backscattering can resolve the liquid-cloud properties around the clock and with narrow temporal resolutions (1-3 $\mathrm{min})$ [5].

\section{METHODOLOGY}

\subsection{Dual-FOV technique for retrievals of liquid- cloud microphysics.}

This lidar-based method makes use of the volume depolarization ratio measured at two FOVs (similarly as the Raman method).

When the light is scattered by cloud droplets, it will be mostly in the forward direction, having a more sharped lobe for large droplets as for small droplets. Forward scattered light may be scattered several further times by different droplets, one wide-angle backscattering event must occur so that it is detected by the receiver telescope. Cross polarized 
light has shown to be sensitive to the droplet size, since in liquid water clouds, depolarization occurs exclusively in multiple scattering regime [5], when the backscattering angle differs slightly from $180^{\circ}$, increasing rapidly as this angle decrease. In the other hand, the larger the FOV, the larger the possible backscattering angles that they detect, so it can be expected that for large droplets, characterized by a sharp forward scattering lobe, the measured depolarization at the two FOVs will be much similar as for small droplets (Figure 1). How much sensitive are the measurements will depend on the size of the FOVs sizes and also the altitude of the cloud base [6].

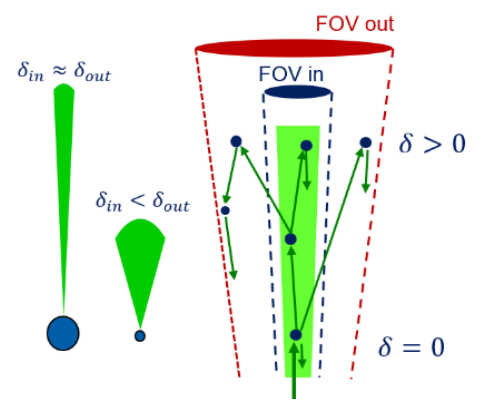

Figure 1: Schematic view of the multiple scattering in cloud droplets.

To quantify this dependence, Simulations of the MS-affected co- and cross-polarized lidar returns at two FOVs were perform. The cloud has been assumed as adiabatic, having a constant increase in the liquid water content with height from the cloud base, while the cloud droplet number concentration remains constant. In this study the FOVs used are $F O V_{\text {in }}=1.0$ and $F O V_{\text {out }}=2.0$ mrads, for a laser with 0.2 mrads divergence. Computations of the volume depolarization ratio at the FOVs "in" and "out" for a wide range of cloud scenes were carried out [7]. The cloud scenes are the vertical profiles of extinction and effective radius, for different reference values and cloud base altitude.

For the retrieval of the cloud parameters, the mean depolarization ratios are calculated $\left(\bar{\delta}_{\text {in }}, \bar{\delta}_{\text {out }}\right)$, and then the ratio between them, we define this term as the relative depolarization: $\delta_{r}=\bar{\delta}_{\text {in }}, / \bar{\delta}_{\text {out }}$,

With the relative depolarization the profile of effective radius can be estimated by using precomputed lookup tables. The penetration depth until which the volume depolarization ratios are averaged was set to 75 meters, which offers the best compromise between measurement uncertainty and sensitivity to the droplet size. For the retrieval of the droplet extinction coefficient we make use of the single-FOV depolarization ratio $\bar{\delta}_{i n}$, and the retrieved effective radius profile. The larger $\delta_{r}$ larger the droplet size, and the larger $\bar{\delta}_{i n}$, larger extinction values. The conversion curves are defined for each cloud base altitude from 1 to $6 \mathrm{~km}$. More details about the computed lookup tables will be available in [8].

\subsection{Implementation of DFOV-Pol technique into PollyXT system for continuous observations.}

In the last two decades, at TROPOS one of the goals has been to develop sophisticated, portable and quality-assured lidar systems (Polly), dedicated to stand-alone operation at remote places [9]. The current setup of the polly system permits continuous observation of the aerosol backscattering at three wavelengths, extinction and depolarization at two wavelengths, and of water vapor mixing ratio profiles.

Recently the Polly system has received the instrumental upgrade to integrate the dual-FOV Polarization retrieval technique into the automated lidar for continuous observations of clouds. The current setup consist of a far-range telescope, with a FOV of 1.0 mrads, equipped with polarization channels (total and cross), calibrated with the $\Delta 90^{\circ}$ method [10]. For the near range a 50mm-diameter telescope is located next to the beam expander, having a FOV of 2.0 mrads. This telescope is coupled by an optical fiber, therefore the polarization components cannot be separated to retrieve the volume depolarization ratio. To achieve this goal, a second $50 \mathrm{~mm}$ telescope was located concentric to the main Telescope, the telescope measures the cross polarized backscatter component and together with the near-range telescope, measuring the total backscatter, they provide the volume depolarization ratio at a second FOV 2.0 mrads [8].

Currently, the polly system, part of the mobile station LACROS (Leipzig Aerosol and Cloud Remote Observations System) is deployed in the city of Punta Arenas, Chile $\left(53^{\circ} \mathrm{S}, 71^{\circ} \mathrm{W}\right)$, for a one-year measurement campaign. The goal is to study the differences between the cloud properties in different extreme environments. Measurements of aerosols and clouds in Leipzig and Punta Arenas, 
offers a unique scenario to study the interaction between aerosols and clouds, contrasting the entanglement in a polluted and in a pristine environment.

\section{RESULTS}

A measurement case to evaluate the interaction between aerosols and clouds in the small scale can visualized in the color map of the Attenuated backscatter at $532 \mathrm{~nm}$ (Fig. 2). The cloud case, with cloud mean cloud base at $1.7 \mathrm{~km}$, has been classified as a liquid layer by the CLOUDNET classification scheme [11]. Figure 3 shows the volume depolarization ratio of the $F O V_{\text {in }}$ in the cloud layer. An increase in the depolarization as MS take place can be observed. Figure $3 b$ presents the so-called relative depolarization ratio. These two observables permits the retrieval of the effective radius and extinction coefficient up to 75 meters in the cloud [8].

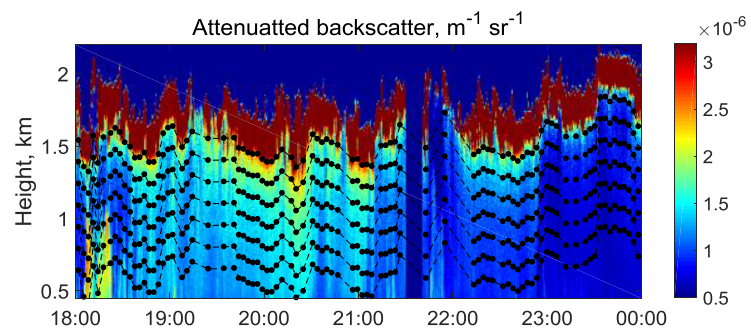

Figure 2: Attenuated backscatter, the black dashed lines describe the aerosol regions considered to evaluate ACI.

The cloud droplet number concentration is then estimated by assuming a modified Gamma distribution with quasy-shape parameter $\mathrm{k}=0.8$ (marine air masses) [Miles 2000]. In order to find the coupled aerosol region, we estimated the so called aerosol cloud interaction index (Eq. 1) diving the region below cloud base in height steps of 150 meters (Figure 2).

As aerosol proxy, we use the aerosol extinction coefficient. Since the measurements were performed at day-time conditions, the retrieval of the backscattering coefficient by using two channels (elastic, Raman $\mathrm{N}_{2}$ ) cannot be afforded. To retrieve this parameter we fixed a constant value for the lidar ratio $L R=25$, the single-channel lidar constant was previously determined in the cloudfree period after the presented case, between 2:00 and 3:00 (not showed). The lidar constant is then used to calculate the attenuated backscatter and the backscattering coefficient is obtained by evaluating the lidar equation so that $\sigma_{a e r}=L R \beta_{a e r}$.

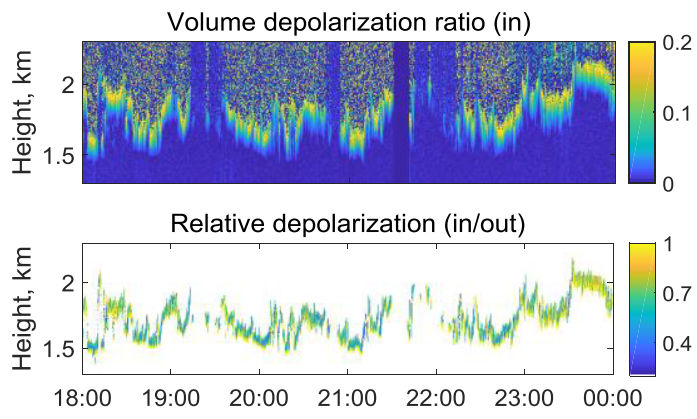

Figure 3: Values of the depolarization $\delta_{i n}$ and ratio of depolarization $\delta_{r}$ used for the retrieval of cloud droplet size and extinction coefficient.

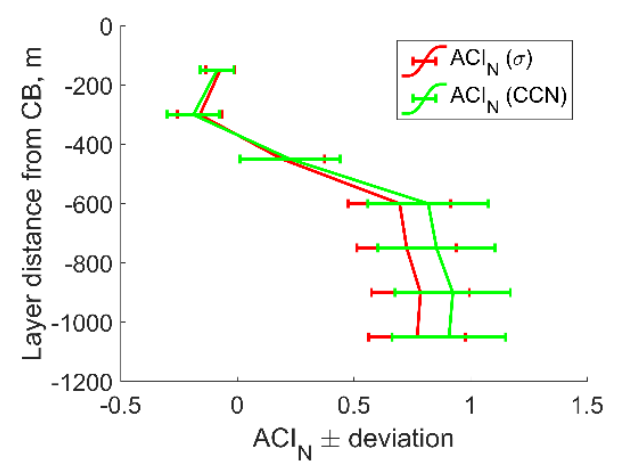

Figure 4: Vertical structure of the Aerosol Cloud Interaction index for each $150 \mathrm{~m}$ layer below cloud base.

To describe the aerosol-cloud interaction based on he observed parameters, the cloud droplet number concentration $C D N C(t)$ and the aerosol extinction coefficient $\sigma(z, t)$ below cloud base, the $A C I_{N}$ was estimated for each layer equidistant to the cloud base during the whole period (Fig. 4). Here it can be noted how the CDNC correlates with the aerosol layers between 600 and 1050 meters, while no correlation is seen in the region right below cloud base, where enhanced aerosol optical properties due to hygroscopic growth mask any correspondence between the measured properties and the CDNC. Then mean values of the extinction in the highly correlated region were used to evaluate the overall cloud response to the aerosol. 
Figure 5 present the time series of the $\mathrm{CDNC}$ and the extinction coefficient of aerosols.

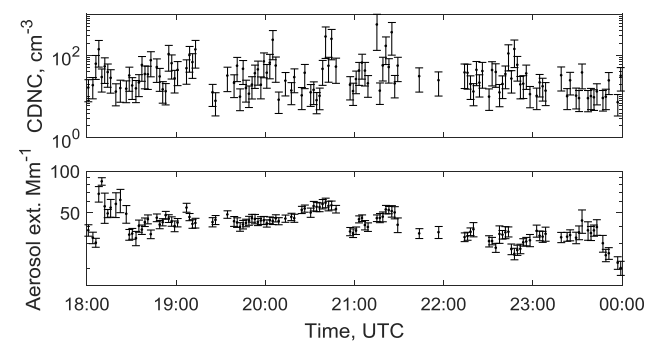

Figure 5: Time series for the CDNC and aerosol extinction with temporal resolution of 2 min.

Figure 6 present the average extinction profile of the 6 hours period. Here, the dashed line is just an indication of the cloud, and does not represent the values of the extinction, since the elastic signal is affected by multiple scattering and the lidar ratio in the cloud $(\sim 18)$ would differ from that of marine aerosols (25). The mean value of the CDNC is 48 $\mathrm{cm}^{-3}$, while the mean extinction value is $41 \mathrm{Mm}^{-1}$, from which we estimated a CCN concentration of $150 \mathrm{~cm}^{-3}$. This results suggests that $1 / 3$ of the aerosols in the layer 600 meters below cloud base has been activated into a cloud droplet.

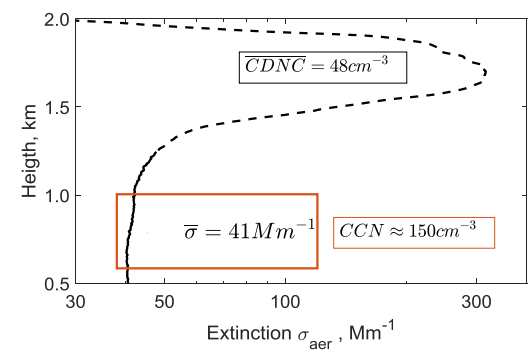

Figure 6: Mean profile of the aerosol extinction coefficient.

The $\mathrm{ACI}_{\mathrm{N}}$, associated to the 2-min observations of the cloud and aerosol parameters (extinction and corresponding $\mathrm{CCN}$ concentrations) are presented in Figures $7 \mathrm{a}$ and $7 \mathrm{~b}$. A linear regression of the data yields to $\quad A C I_{N}(\sigma)=0.76 \pm 0.29$ and $A C I_{N}(C C N)=0.89 \pm 0.37$. The pristine ambient of the region surrounding the city of Punta Arenas, and the aerosol type, identified as marine from backward trajectories presents favorable conditions for aerosol-cloud interactions given the large numbers obtained for its index. These results are also in agreement with [12], where the relation between extinction and $\mathrm{CCN}$ constrains the maximum $\mathrm{ACI}_{\mathrm{N}}$ from extinction observations to 0.8 .
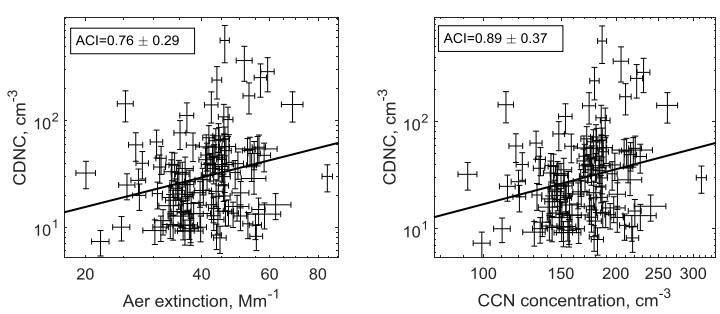

Figure 7: (a) CDNC vs aerosol extinction for the 6 hours period. (b) CDNC vs CCN concentration.

\section{CONCLUSIONS}

This work presents an application of the observation capabilities of a Dual-FOV Polarization system. The 2-min. retrievals of the cloud droplet number concentration and of aerosol extinction coefficient at different layers below cloud base shows how the cloud responds to the aerosol presence in the boundary layer, obtaining large correspondence in the region between 600 to 1105 meters below cloud base $A C I_{N}=0.76 \pm$ 0.29 . Further analysis of more cases will allow us to study aerosol-cloud interactions in different environments (polluted and not-polluted). Vertical movement is another important parameter to consider. Long-term measurements of aerosol and cloud properties, combined with vertical wind information (Doppler Lidar) will permit a deep statistical analysis of the interaction.

\section{REFERENCES}

[1] G. Feingold, J. Geo. Res., 106, 22907-22922 (2001). [2] R. Mamouri, A. Ansmann, Atmos. Chem. Phys., 16, 5905-5931 (2016).

[3] L. Bissonnette, et al., Appl. Phys. B 60, 355-362 (1995).

[4] J. Schmidt, et al., Appl. Opt., 52, 2235-2247 (2013).

[5] I. Veselovskii, et al., Appl. Opt. 45, 6839-6848 (2006)

[6] C. Jimenez, et al., Proc. SPIE, 10429, 1042907 (2017)

[7] E. Zege, et al., Appl. Phys. B 60, 345-353 (1995).

[8] C. Jimenez, et al., Atnos, Meas. Tech, (in preparation, 2020).

[9] R. Engelmann, et al., Atmos. Meas. Tech., 9, 17671784 (2016)

[10] V. Freudenthaler, Atmos. Meas. Tech., 9, 41814255 (2016).

[11] H. Baars, et al., Atmos. Meas. Tech., 10, 31753201 (2017).

[12] Y. Shinozuka, et al., Atmos. Chem. Phys., 15, 7585-7604 (2015) 